\title{
Metal-organic framework thin films on a surface of optical fibre long period grating for chemical sensing
}

\author{
J. Hromadka ${ }^{* a, b}$, B. Tokay ${ }^{\mathrm{c}}$, S. James ${ }^{\mathrm{d}}$, S. Korposh ${ }^{\mathrm{a}, \mathrm{d}}$ \\ ${ }^{a}$ Optics \& Photonics Group, Electrical Systems and Optics Research Division, Faculty of \\ Engineering, University of Nottingham, University Park, Nottingham NG7 2RD, UK \\ ${ }^{\mathrm{b}}$ Institute for Environmental Studies, Faculty of Sciences, Charles University in Prague, Benátská 2, \\ CZ 12843 Praha 2, Czech Republic \\ ${ }^{c}$ Chemical and Environmental Engineering Department, Faculty of Engineering, University of \\ Nottingham, University Park, Nottingham NG7 2RD, UK \\ ${ }^{\mathrm{d}}$ Engineering Photonics, Cranfield University, Cranfield, Bedfordshire MK43 0AL, UK
}

\begin{abstract}
An optical fibre long period grating (LPG) modified with a thin film of HKUST-1, a material from metal organic framework (MOF) family, was employed for the detection of carbon dioxide. The sensing mechanism is based on the measurement of the change of the refractive index (RI) of the coating that is induced by the penetration of $\mathrm{CO}_{2}$ molecules into the HKUST-1 pores. The responses of the resonance bands in the transmission spectrum of an LPG modified with 40 layers of HKUST-1 upon exposure to carbon dioxide in mixture with nitrogen were investigated.
\end{abstract}

Keywords: Long period grating (LPG), metal organic framework (MOF), HKUST-1, layer by layer deposition, carbon dioxide $\left(\mathrm{CO}_{2}\right)$

\section{INTRODUCTION}

Although numerous chemical sensors already exist, there is still a requirement for the development of new sensing technologies. New sensors could target demand in areas such as medical diagnostics, food quality control, air quality (occupational exposure) and in various fields of environmental monitoring ${ }^{1}$. The development of low-cost, portable, precise and real-time sensors for the detection of carbon oxides, organic vapours such as methane and volatile organic compounds is of significant interest. The key element of any portable sensor is the sensitive layer that captures the analyte gases.

Metal organic frameworks (MOFs) offer an ideal platform for the development of sensitive films with responses to specific analytes. They can be considered as crystalline materials with tuneable porosity, large internal surface area and organic functionality. The strong metal-oxygen-carbon bonds endow the materials with high chemical and thermal stabilities $^{2}$. The adjustment of the pore size and possibility of post-synthesis functionalization enable the specific reaction of the selected type of MOF with the analyte of interest, where only certain molecules (defined by size or functional group) can penetrate into the MOF's cavities.

There have been limited reports of MOF based sensors, but they indicate their potential to become powerful analytical devices $^{1}$. The main advantage is the high chemical selectivity made possible by the appropriate selection of a MOF with desired properties ${ }^{3}$. However, there is a need to establish a suitable means of signal transduction to enable the use of MOFs for chemical sensing ${ }^{4}$. The pores of MOF structures are too small to incorporate reporting molecules. The most promising approach appears to be the adoption of a macroscopic perspective and to analyse changes in the properties of the whole film. This can be achieved by the use of an optical transduction technique, which requires the fabrication of a film, ideally with controllable thickness, on an optical sensing platform to develop a chemically sensitive interferometer ${ }^{4}$. We have recently reported a fibre optic long period grating (LPG) based MOF sensor for the detection of organic vapours, where ZIF-8 had been deposited on the surface of the LPG with use of an in-situ crystallization technique ${ }^{5}$.

*jiri.hromadka@nottingham.ac.uk; phone+44-0115-8467892

This paper was published in 25th International Conference on Optical Fiber Sensors and is made available with permission of SPIE. One print or electronic copy may be made for personal use only. Systematic electronic or print reproduction and distribution, duplication of any material in this paper for a fee or for commercial purposes, or modification of the content of the paper are prohibited. 
Different methodologies have been used for the fabrication of MOF thin films, including solvothermal synthesis, microwave induced thermal deposition, seeded growth and in-situ crystallization ${ }^{6}$. This work will investigate the use of the layer-by-layer technique, which involved the deposition of oppositely charged materials that can be added to the fibre on the molecular level to build up a coating of the required thickness. The entire coating can be made from the sensitive material and then react with the compound of interest, leading to a change of refractive index ${ }^{7}$.

The layer by layer deposition has been used previously for the fabrication of MOF films ${ }^{8,9}$ and its use is desired due to the simplicity of the procedure, which is conducted at room temperature, requires less time and involves minimal use of consumables in comparison to other techniques. In particular, for HKUST-1 the LbL approach leads to the fabrication of films with higher crystal density, conforming into homogenous films with preferred [222] orientation?. Among the variety of MOFs, thin films of HKUST-1 are considered to be highly selective for carbon dioxide sensing due to the presence of unsaturated copper ${ }^{(I I)}$ metal centres and due to its structure, which consists of two large central cavities $(9 \AA)$ surrounded by smaller $5 \AA$ cavities $^{10}$.

An LPG consists of a periodic perturbation of the refractive index of the core, which couples the core mode to a set of co-propagating modes of the cladding of the fibre ${ }^{11}$ and it has been widely used as a platform for the various range of sensors ${ }^{7,11,12}$.

In this work, for the first time to the best of our knowledge, we present the deposition of HKUST-1, a material from MOF family, as a thin film on the surface of an LPG using layer by layer technique. The functionalized LPG was subsequently tested for its sensitivity to carbon dioxide.

\section{EXPERIMENT}

An LPG with a grating period of $109.0 \mu \mathrm{m}$ and length of $30 \mathrm{~mm}$ was fabricated in boron-germanium co-doped optical fibre (Fibercore PS750) with the cut-off wavelength of $670 \mathrm{~nm}$ in a point-by-point fashion, side-illuminating the optical fibre by the output from a frequency-quadrupled Nd:YAG laser operating at $266 \mathrm{~nm}^{13}$. The transmission spectrum of the optical fibre was recorded by coupling the output from a tungsten-halogen lamp (Ocean Optics HL-2000) into the fibre, analysing the transmitted light using a fibre coupled CCD spectrometer (Ocean Optics HR4000). The grating period was selected such that the LPG operated at or near the phase matching turning point ${ }^{14}$, which, for coupling to a particular cladding mode (in this case $\mathrm{LP}_{019}$ ), ensured optimized sensitivity.

The LPG was coated with HKUST-1 using a layer by layer technique ${ }^{9}$. Briefly, solutions of $1 \mathrm{mM} 1,3,5-$ benzenetricarboxylic acid $\left(\mathrm{H}_{3}\right.$ btc $)$ and $0.2 \mathrm{mM}$ copper acetate $\left(\mathrm{Cu}_{2}\left(\mathrm{AcO}_{4}\right)\right.$ in ethanol were prepared. The LPG was fixed in a specially designed holder to keep the LPG taut and straight. The LPG was placed inside a container and immersed in a $1 \mathrm{wt} \% \mathrm{KOH}$ in ethanol/water $=3: 2 \mathrm{v} / \mathrm{v}$ solution for $20 \mathrm{~min}$, leading to a negatively charged surface. The optical fibre was then sequentially immersed into a $\left(\mathrm{Cu}_{2}\left(\mathrm{AcO}_{4}\right)\right.$ solution and an $(\mathrm{H} 3 \mathrm{btc})$ solution for $5 \mathrm{~min}$ each, resulting in the alternate deposition of $\mathrm{Cu}^{2+}$ and btc', forming an HKUST-1 film on the surface of LPG. Between each of these steps the LPG was washed by ethanol using a plastic pipette to remove all unreacted parts of film forming solutions. The process was repeated to obtain a thicker film consisting of 40 layers. Transmission spectra were recorded continuously during the deposition process. The performance of the LPG as a chemical sensor was investigated by exposing the coated device to elevated concentrations of carbon dioxide, up to 40,000 ppm. The LPG was fixed inside the closed container and kept taut and straight. The nitrogen flow was introduced into the chamber followed by carbon dioxide until it reaches concentration levels of $\sim 10,000,20,000$ and 40,000 and the stable conditions were kept for 20 min at each step.

The central wavelengths of the resonance bands in the LPG's transmission spectra were recorded and their response to the presence of carbon dioxide was evaluated. The concentration of carbon dioxide, the temperature and the relative humidity (RH) were recorded by a data logger (K-33 ICB 30\% $\mathrm{CO}_{2}$ sensor from $\mathrm{CO} 2$ meter, Inc.) An acquisition interval of $20 \mathrm{~s}$ was set for all experiments.

\section{RESULTS AND DISCUSSIONS}

The transmission spectrum of an LPG with period of $109.0 \mu \mathrm{m}$, recorded before and after the deposition process, is shown in Figure 1a (black and blue line respectively). A resonance band near the PMTP of the $\mathrm{LP}_{019}$ cladding mode is observed in the region of $850 \mathrm{~nm}$ and it splits into two bands after the deposition. The band at around $680 \mathrm{~nm}$ corresponds to coupling to a lower order cladding mode $\left(\mathrm{LP}_{018}\right)^{11,14}$. 
The evolution of the attenuation band at the PMTP can be observed in the transmission spectrum recorded with the LPG in air after the deposition of 20 layers of HKUST-1, Figure 1a. When the spectrum was measured with the LPG immersed in methanol, a continuous change in the transmission spectra with increasing coating thickness was observed. The attenuation band at the PMTP splits into two after the deposition of the HKUST-1 film, where the transmission spectra were measured in air, Figure 1a. The central wavelength difference of the resonance bands corresponding to coupling to the $\mathrm{LP}_{019}$ cladding mode $\left(\mathrm{CW}_{\text {diff LP019}}\right)$ exhibits a linear dependence upon the number of deposited layers, suggesting that the thickness of the thin film increases linearly with the increasing number of layers, Figure $1 \mathrm{~b}$.

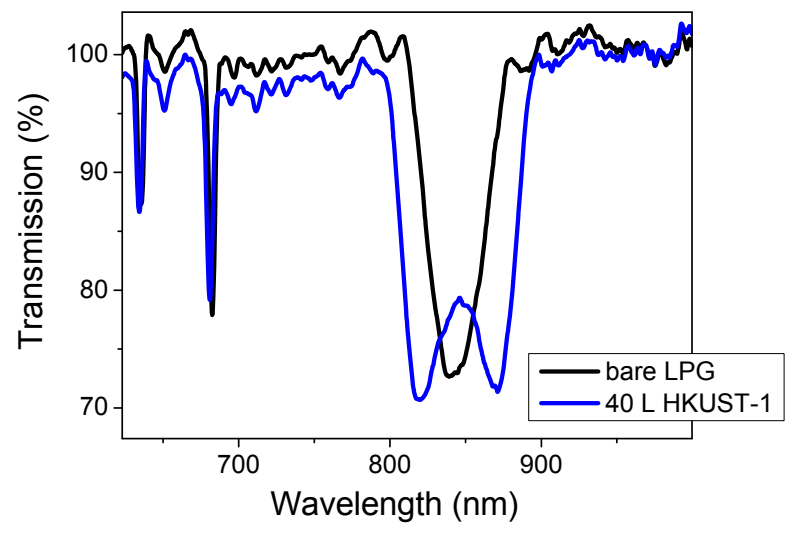

(a)

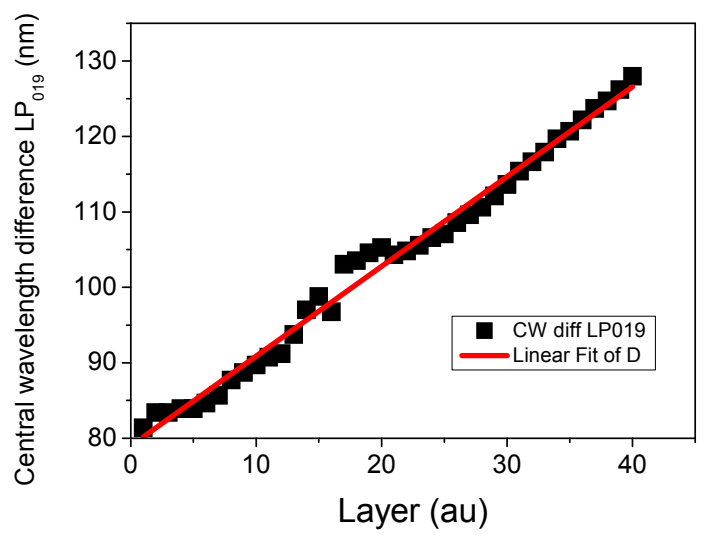

(b)

Figure 1. (a) Transmission spectra of the LPG with period of $109.0 \mu \mathrm{m}$ measured in air with attenuation bands at 830,690 and $640 \mathrm{~nm}$ corresponding to $\mathrm{LP}_{019}, \mathrm{LP}_{018}$ and $\mathrm{LP}_{017}$ cladding modes; black line, uncoated LPG, blue line, after the deposition of 40 layers of HKUST-1; b) change in the position of the central wavelength corresponding to LP $_{018}$ cladding mode during the deposition of $1^{\text {st }}$ to $40^{\text {th }}$ layer of HKUST-1.

When exposed to carbon dioxide, the continuous shift of $\mathrm{CW}_{\text {diff LP019 }}$ was observed over the whole concentration range up to $41,200 \mathrm{ppm}$, where it reached $10.58 \mathrm{~nm}$ (9.64 nm after compensating for the influence of temperature) (Figure 2).

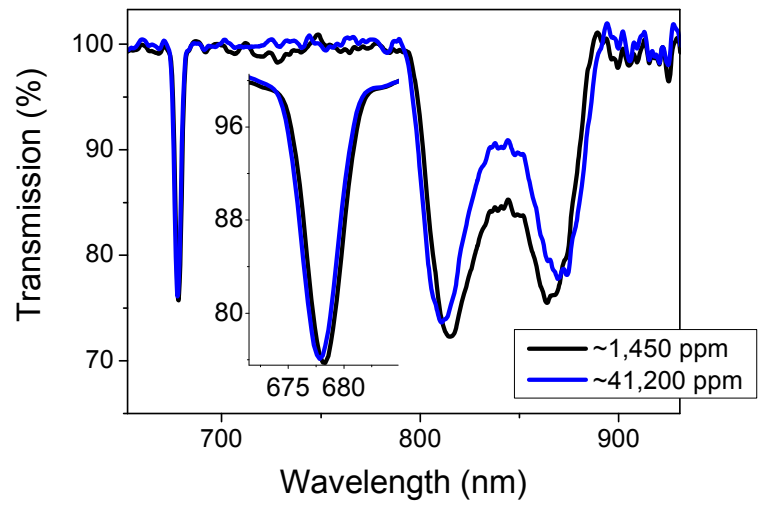

(a)

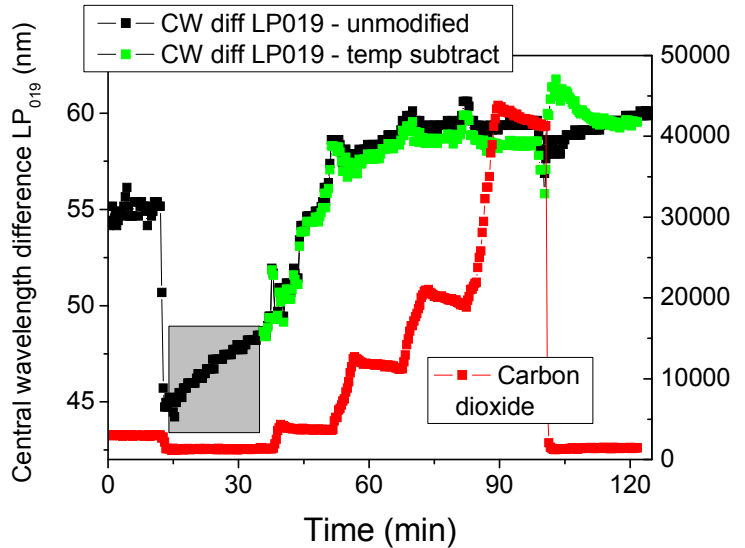

(b)

Figure 2. The sensor coated with 40 layers of HKUST-1 exposed to carbon dioxide: a) Transmission spectra at 1,450 (black) and 41,200 ppm of carbon dioxide (blue); the inset shows the attenuation band corresponding to the $\mathrm{LP}_{018}$ cladding mode in detail and b) dynamic shift of the central wavelength difference before (black) and after the correction for the influence of temperature (green); concentration of carbon dioxide (red).

The influence of temperature on the sensor performance was characterised by keeping the $\mathrm{CO}_{2}$ level constant while increasing the temperature (data shown in the grey box in Figure 2a). This data allowed the temperature compensation of 
measurements of $\mathrm{CW}_{\text {diff LP019 }}$. The response of the sensor was observed to be irreversible as no change in the transmission spectrum was detected when the chamber was flushed with nitrogen and the carbon dioxide concentration returned to levels close to $1,300 \mathrm{ppm}$.

The thorough study on the deformation of the film by ambient RH needs to be included in the future work, as it is known that water vapour can react with the open copper sites in the HKUST-1 structure and then block their specific affinity to carbon dioxide molecules. This effect was previously described for degradation with time of a HKUST-1 film stored under elevated relative humidity levels 9 .

\section{CONCLUSIONS}

An HKUST-1 film, a material from metal organic framework family, has been deposited successfully onto the surface of an LPG using for the layer-by layer technique. The linear shift of the central wavelength during the deposition indicates the uniform growth of the HKUST-1 film with the increasing number of layers. The LPG coated with 40 growth cycles of HKUST-1 exhibited sensitivity to carbon dioxide. Future work will include the further characterization of the HKUST-1 film and an investigation of the cross sensitivity to other analytes.

\section{REFERENCES}

[1] Kreno, L. Leong, K., Farha, O., Allendorf, M., Van Duyne, R. and Hupp, J. "Metal-organic framework materials as chemical sensors," Chemical Reviews, 112(2), 1105-1125 (2012).

[2] Yaghi, O., O'Keeffe, M., Ockwig, N., Chae, H., Eddaoudi, M. and Kim, J. "Reticular synthesis and the design of new materials," Nature, 423(6941), 705-714 (2003).

[3] Kuppler, R., Timmons, D., Fang, Q.-R., Li, J.-R., Makal, T. A., Young, M. D. Yuana, D., Zhaoa, D. and Zhuang, W. Z. H.-C. "Potential applications of metal-organic frameworks," Coordination Chemistry Reviews, 253(23-24), 3042-3066 (2009).

[4] Lu, G. and Hupp, J. "Metal-organic frameworks as sensors: A ZIF-8 based fabry-pérot device as a selective sensor for chemical vapors and gases," Journal of the American Chemical Society, 132(23), 7832-7833 (2010).

[5] Hromadka, J., Tokay, B., James, S., Tatam, R. P., Korposh, S. "Optical fibre long period grating gas sensor modified with metal organic framework thin films," Sensors and Actuators, B: Chemical, 221, 891-899 (2015).

[6] Bétard, A. and Fischer, R. A. "Metal-organic framework thin films: From fundamentals to applications," Chemical Reviews, 112(2), 1055-1083 (2012).

[7] Korposh, S., Selyanchyn, R., Yasukochi, W., Lee, S.-W., James, S. and Tatam, R."Optical fibre long period grating with a nanoporous coating formed from silica nanoparticles for ammonia sensing in water," Materials Chemistry and Physics, 173, 784-792 (2012).

[8] So, M. C., Jin, S., Son, H.-J., Wiederrecht, G. P., Farha, O. K. and Hupp, J. T."Layer-by-layer fabrication of oriented porous thin films based on porphyrin-containing metal-organic frameworks," Journal of the American Chemical Society, 135(42),15698-15701 (2013).

[9] Nijem, N., Fürsich, K., Kelly, S. T., Swain, C., Leone, S. R. and Gilles, M. K. "HKUST-1 thin film layer-by-layer liquid phase epitaxial growth: film properties and stability dependence on layer number," Crystal Growth and Design, 15(6), 2948-2957 (2015).

[10] Gutiérrez-Sevillano, J. J., Vicent-Luna, J. M., Dubbeldam, D. and Calero, S. "Molecular mechanisms for adsorption in Cu-BTC metal organic framework," Journal of Physical Chemistry C, 34(3), 11357-11366 (2013).

[11] James, S. and Tatam, R. "Optical fibre long-period grating sensors: characteristics and application," Meas Sci Technol R49-R61 (2003).

[12] Rego, G. "A review of refractometric sensors based on long period fibre gratings," Scientific World J, 913418 (2013).

[13] Hromadka, J., Correia, R. and Korposh, S. "Fabrication of fiber optic long period gratings operating at the phase matching turning point using an amplitude mask, Proceedings of the Sixth European Workshop on Optical Fibre Sensors, Limerick, Ireland, paper ID 9916, 99160Y

[14] Wong, R. Y. N., Chehura, E., Staines, S. E., James, S. W. and Tatam, R. P. "Fabrication of fiber optic long period gratings operating at the phase matching turning point using an ultraviolet laser," Applied Optics, 53(21), 4669-4674 (2014). 
2017-04-23

\section{Metal-organic framework thin films on a surface of optical fibre long period grating for chemical sensing}

Hromadka, J.

SPIE

J. Hromadka; B. Tokay; S. James; S. Korposh. Metal-organic framework thin films on a surface of optical fibre long period grating for chemical sensing. 25th International Conference on Optical Fiber Sensors, OFS 2017; 24-28 April 2017, Jeju; South Korea. Paper number: 103234C https://dspace.lib.cranfield.ac.uk/handle/1826/12738

Downloaded from Cranfield Library Services E-Repository 